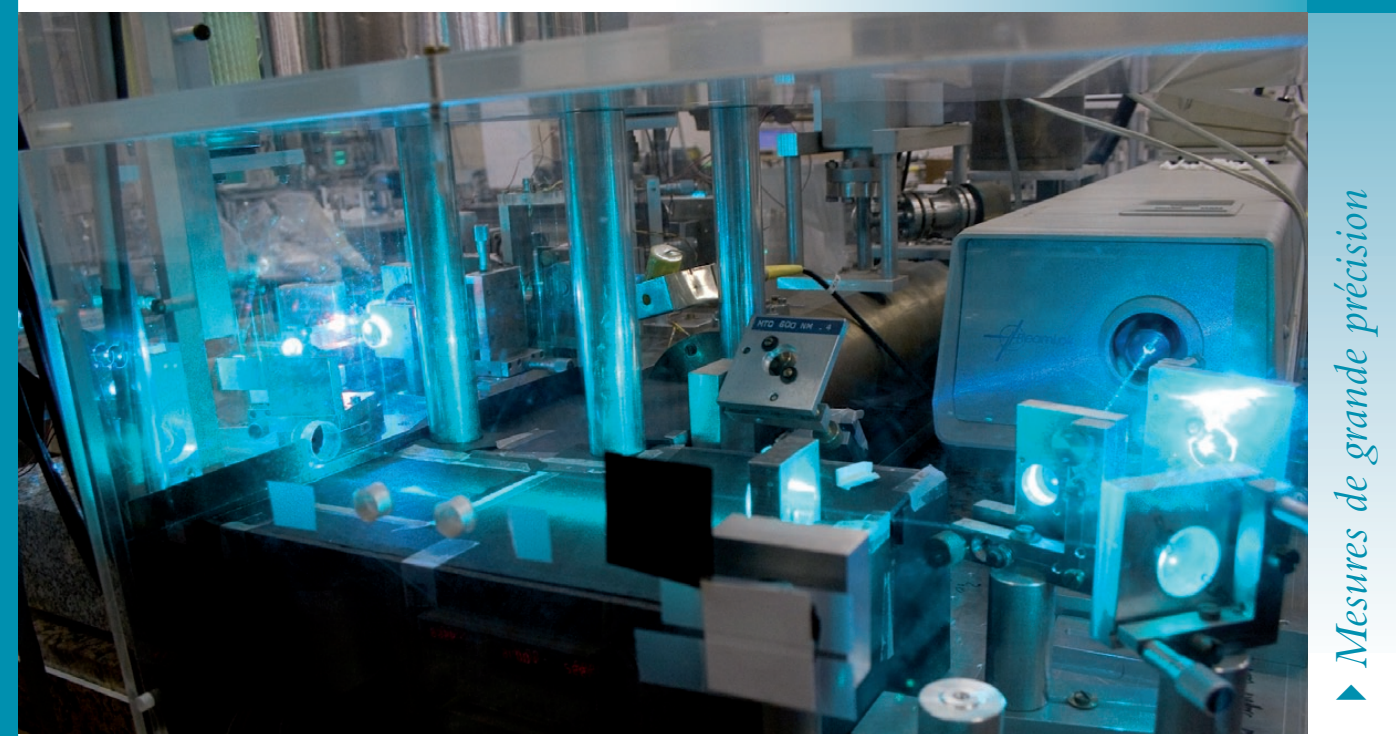

Système optique pour le pompage d’un laser titane-saphir, utilisé au LKB pour la détermination de la constante de structure fine, en mesurant la vitesse de recul d'atomes ultra-froids. @ Jean-François DARS

\title{
Le laser : un outil de choix pour l'interférométrie atomique
}

Arnaud Landragin et Franck Pereira Dos Santos (franck.pereira@obspm.fr)

Systèmes de référence temps-espace (SYRTE), CNRS et UPMC, Observatoire de Paris, 61 avenue de l'Observatoire, 75014 Paris

Les lasers sont un élément

clé dans de nombreuses

expériences $d^{\prime}$ 'interférométrie

atomique, parce qu'ils

permettent de manipuler

de façon remarquablement

contrôlée les ondes de

de Broglie associées

aux atomes. Ils sont exploités notamment pour réaliser des

capteurs inertiels atomiques

- accéléromètres et gyromè-

tres - de très haute sensibilité.

Au-delà de la navigation,

ces instruments possèdent

de nombreux champs

d'application, tels que

la géophysique, la métrologie

ou encore la physique

fondamentale.

\section{Historique}

L'interférométrie atomique exploite la nature ondulatoire de la matière. Si chacun est familier avec le caractère ondulatoire de la lumière, qui se manifeste de façon spectaculaire au travers d'interférences en optique, imaginer des phénomènes analogues avec des objets matériels est plus difficile à concevoir pour beaucoup. Pourtant, la nature duale de la matière a été énoncée il y a près d'un siècle, à l'époque du développement de la physique quantique, par Louis de Broglie, qui a proposé d'associer aux particules et aux atomes des propriétés ondulatoires, caractérisées par la longueur d'onde dite de de Broglie ; celle-ci est donnée par $\Lambda_{d B}=h / p$, où $h$ est la constante de Planck et $p$ l'impulsion de la particule, de l'atome ou de la molécule. Pour les atomes émis depuis un four dans un jet effusif (typiquement à quelques centaines de degrés Celsius), $\Lambda_{d B}$ est de l'ordre d'une dizaine de picomètres ${ }^{(1)}$, ce qui est beaucoup plus faible que la longueur d'onde du rayonnement électromagnétique dans le visible (qui est de l'ordre du micromètre). On conçoit bien, dès lors, que ces propriétés ondulatoires ont été plus difficiles à mettre en évidence, et qu'il a fallu développer des outils spécifiques pour manipuler les ondes de matière.
Plusieurs expériences, analogues aux expériences de diffraction en optique, ont néanmoins été réalisées peu après. Elles ont montré le caractère ondulatoire de la matière, avec des particules élémentaires d'abord (diffraction d'électrons en 1927, de neutrons en $1945 \ldots$..), puis avec des atomes (réflexion d'atomes d'hélium sur une surface cristalline). Mais il a fallu attendre la fin du $\mathrm{xx}^{\mathrm{e}}$ siècle, et l'avènement des nanotechnologies, pour parvenir à fabriquer des objets matériels à l'aide desquels on peut faire diffracter des ondes de matière. Ces objets présentent en effet des structures (trous, fentes...) de très petite taille (de l'ordre de $100 \mathrm{~nm}$ ), bien plus faible, par exemple, que la longueur de cohérence transverse d'atomes dans un jet atomique très bien collimaté (qui peut atteindre plusieurs micromètres). Des réseaux de transmission nanofabriqués, constitués de fentes de $200 \mathrm{~nm}$, ont ainsi été utilisés pour réaliser d'abord des expériences de diffraction, puis pour créer, à l'aide de trois réseaux de ce type, un des tout premiers interféromètres atomiques en 1991.

Cette même année, les premiers interféromètres atomiques utilisant des lasers ont également été démontrés, et se sont rapidement révélés plus performants. 

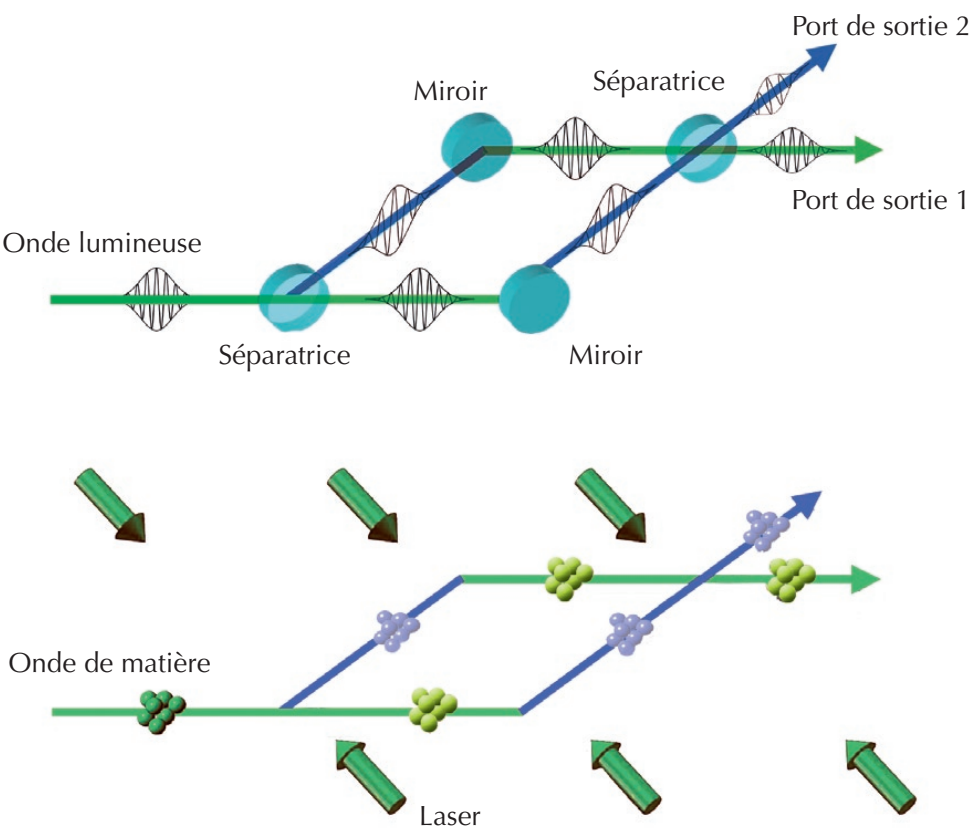

1. Analogie entre un interféromètre optique de type Mach-Zehnder (a) et un interféromètre atomique (b), dans lequel les atomes sont placés dans une superposition quantique de deux trajectoires séparées spatialement puis recombinées. Dans l'interféromètre atomique, les fonctions analogues aux séparatrices et miroirs de l'interféromètre optique peuvent être réalisées à l'aide de brèves impulsions laser (ici au nombre de trois, voir l'encadré 1). Entre les impulsions lasers, les paquets d'ondes voyagent librement. Les chemins correspondant aux deux états d'impulsion sont représentés par des traits de couleurs différentes.

\section{Diffraction d'une onde atomique sur une onde stationnaire}

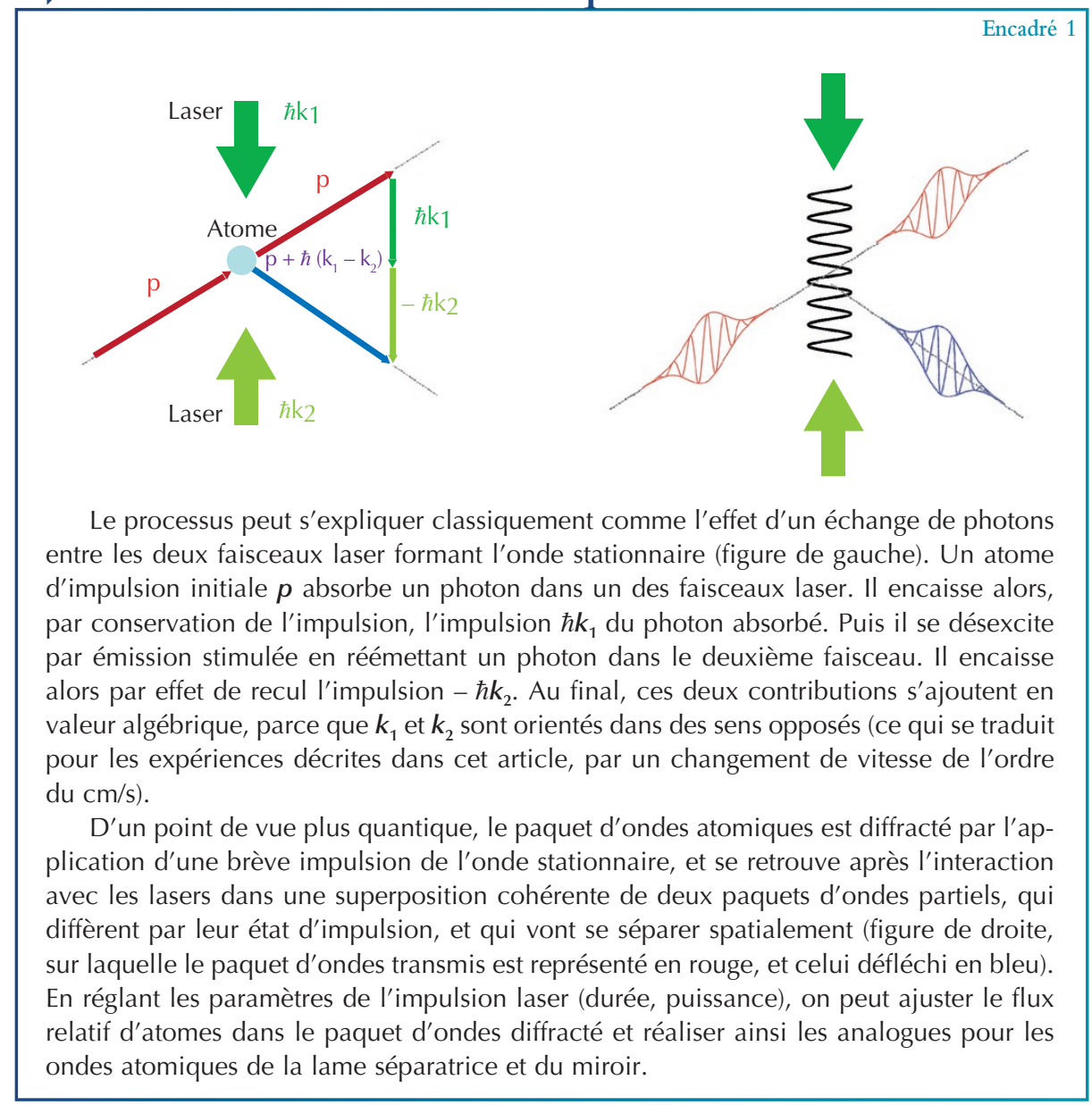

$\gg>$

\section{Des lasers pour manipuler les paquets d'ondes atomiques}

L'outil de choix pour manipuler les ondes de matière, c'est le laser. Ici, les propriétés intrinsèques du laser (directivité, monochromaticité, possibilité d'en déterminer la longueur d'onde avec exactitude), ainsi que la compréhension fine de l'interaction matière-rayonnement, sont les éléments clés pour une manipulation parfaitement contrôlée des ondes de matière. Par exemple, une onde stationnaire, obtenue par la rétroréflexion d'un faisceau laser sur luimême, crée pour les ondes de matière un milieu analogue à un réseau d'indices, dans lequel le potentiel d'interaction atome-laser est modulé spatialement et sur lequel les ondes de matière diffractent. Cette diffraction est liée à des processus d'absorption et d'émission stimulée de photons dans l'onde stationnaire (encadré 1). En effet, lorsqu'un atome absorbe (ou émet) un photon, il acquiert (ou cède) non seulement l'énergie du photon, mais aussi son impulsion, ce qui permet de le défléchir. Du point de vue quantique, l'interaction avec les lasers permet alors de placer l'onde atomique dans une superposition de deux paquets d'ondes partiels, qui se séparent spatialement.

Comment réaliser un interféromètre atomique ? On peut exploiter l'analogie avec les ondes lumineuses et concevoir, par exemple, un interféromètre de type Mach-Zehnder (fig. 1). Dans sa version optique, cet interféromètre est constitué d'une première lame séparatrice qui permet de diviser l'onde lumineuse, puis de deux miroirs qui redirigent et recombinent les ondes lumineuses sur une seconde lame séparatrice. L'intensité dans chacun des deux ports de sortie est alors modulée en fonction de la différence de phase accumulée le long des deux chemins optiques. Dans la version "matérielle " de l'interféromètre, les paquets d'ondes atomiques sont séparés, redirigés et recombinés à l'aide d'éléments jouant le rôle des séparatrices pour les ondes de matière, et qui sont réalisés avec des objets matériels (comme des réseaux nanofabriqués) ou avec des faisceaux lasers (comme l'onde stationnaire décrite ci-dessus). Dans tous les cas, les deux ports de sortie correspondent à des états d'impulsion différents. Le déphasage en sortie d'interféromètre s'obtient alors en mesurant les flux d'atomes dans les deux ports de sortie, qui sont modulés en fonction de la 
différence des phases accumulées par les ondes de matière dans deux bras de l'interféromètre. Cette géométrie d'interféromètre est notamment celle des capteurs inertiels atomiques, que nous allons décrire ci-dessous.

\section{Mesure des forces d'inertie}

Les capteurs inertiels sont des instruments couramment utilisés pour mesurer les accélérations et les vitesses de rotation des engins mobiles sur lesquels ils sont embarqués. Un interféromètre de MachZehnder atomique est sensible également aux accélérations et aux rotations. En effet, la différence de phase accumulée par les ondes de matière le long des deux bras de l'interféromètre est proportionnelle simultanément à l'accélération le long des faisceaux lasers, et à la vitesse de rotation autour d'un axe perpendiculaire à l'aire orientée, définie par les deux bras de l'interféromètre ${ }^{(2)}$. Pour une durée passée par les atomes dans l'interféromètre de l'ordre d'une centaine de millisecondes, qu'il est possible d'obtenir en utilisant des atomes refroidis par lasers [1], ces déphasages sont très grands (plusieurs centaines de milliers de radians pour une accélération de l'ordre de $g$, et quelques radians pour une vitesse de rotation aussi faible que celle de la rotation de la Terre).

Dans la plupart des expériences de capteurs inertiels atomiques, les atomes alcalins utilisés (césium ou rubidium) interagissent non pas avec une onde stationnaire fixe, mais avec une onde stationnaire en mouvement, réalisée à l'aide de deux faisceaux lasers se propageant en sens contraires et de fréquences légèrement différentes. Ceci permet d'associer au changement d'impulsion de l'atome un changement de son état interne (on parle de transitions Raman stimulées entre les sous-niveaux hyperfins de l'atome). On peut ainsi réaliser un interféromètre à trois impulsions, similaire à un Mach-Zehnder, à la sortie duquel on détecte en fait l'état électronique de l'atome par une mesure de fluorescence [2].

Dans cette configuration, la sensibilité aux forces d'inertie provient de l'interaction avec les lasers. Lors des impulsions Raman, la différence de phase entre les faisceaux laser s'imprime sur les paquets d'ondes atomiques diffractés. Le déphasage dépend donc des positions des paquets d'ondes atomiques, qui sont modifiées par les forces d'inertie, par rapport aux plans d'égale différence de phase des lasers, qui sont liés au référentiel du laboratoire. La différence de phase atomique en sortie de l'interféromètre s'exprime simplement comme une combinaison linéaire des différences de phase laser vues par les atomes à chacune des impulsions. Finalement, les lasers jouent ici le rôle d'une règle extrêmement précise pour mesurer ces déplacements, grâce à la bonne connaissance de la longueur d'onde du laser, qui garantit l'exactitude de la mesure.

Dans le cas d'une accélération, la sensibilité de l'interféromètre croît comme le déplacement des atomes suivant la direction des faisceaux laser, et est donc proportionnelle au carré du temps de vol entre la première et la dernière impulsion. Dans le cas d'une rotation, le déphasage est proportionnel à l'aire physique, c'est-à-dire au produit de la longueur par la séparation spatiale des bras ; la sensibilité est donc proportionnelle à la vitesse des atomes dans le plan orthogonal aux faisceaux laser et, comme pour l'accélération, au carré du temps de vol. Suivant la géométrie de l'interféromètre (l'orientation des faisceaux laser par rapport à la trajectoire des atomes), on peut réaliser des accéléromètres, des gyromètres ou des instruments permettant les deux types de mesures (fig. 2). Par exemple, lorsque les lasers sont orientés le long de la verticale, l'aire physique est nulle et l'interféromètre mesure uniquement l'accélération de la pesanteur.

\section{Mesures en métrologie fondamentale}

Grâce au caractère exact de leurs mesures, les interféromètres atomiques sont d'excellents outils pour la métrologie fondamentale. La première de ces applications concerne la mesure directe de la constante de structure fine $\alpha\left(=e^{2} / h c \approx 1 / 137\right)$, qui régit la force de couplage entre les photons et les électrons [4]. Dans ces expériences, un interféromètre est utilisé pour mesurer la vitesse de recul des atomes, après un cycle d'absorption-émission stimulée dans les séparatrices atomiques. En pratique, ces expériences mesurent l'effet Doppler lié au changement de vitesse après un grand nombre de ces cycles, ce qui permet d'augmenter la sensibilité de la mesure. Grâce à la connaissance précise de la longueur d'onde des lasers utilisés pour réaliser et mesurer ce changement de vitesse, on

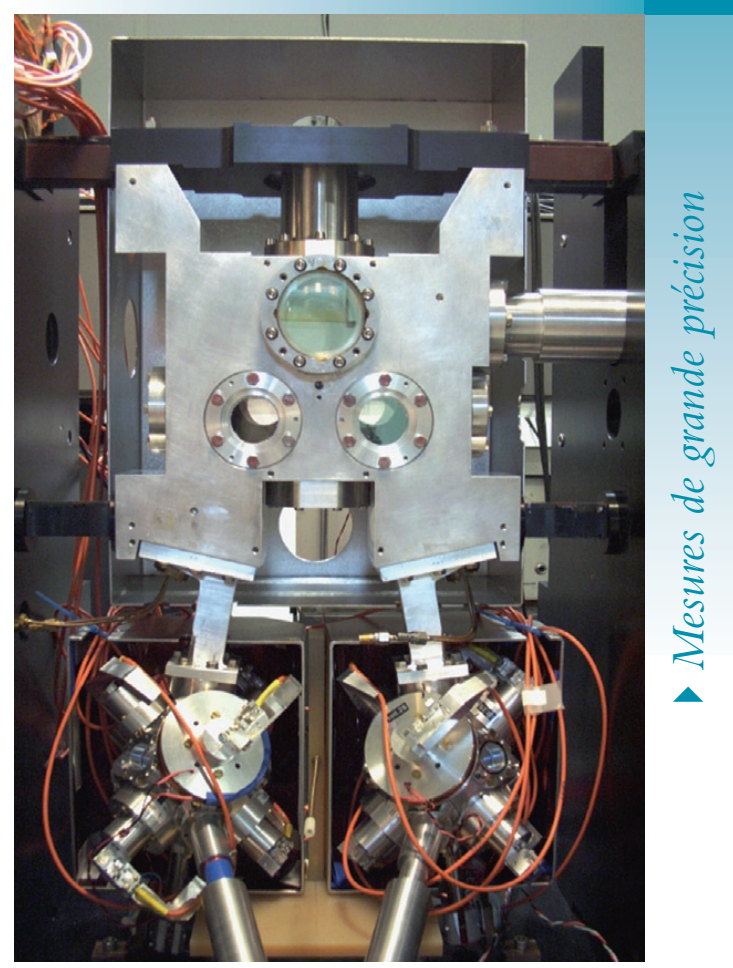

2. Capteur inertiel ayant la possibilité de mesurer les accélérations et les vitesses de rotation suivant tous les axes d'inertie dans un même instrument $\left(30 \times 50 \mathrm{~cm}^{2}\right)$ [3]. Ce dispositif utilise deux boules d'atomes froids lancées l'une vers l'autre, vers le haut et selon une trajectoire parabolique. À l’apogée de la trajectoire, on réalise un double interféromètre, en utilisant les mêmes impulsions laser sur les deux boules simultanément. Si chaque interféromètre est sensible à la fois à l'accélération et à la vitesse de rotation, on peut séparer les déphasages d'accélération et de rotation en combinant les mesures des deux instruments.

déduit de la mesure de vitesse de recul le rapport $h / M$, où $M$ est la masse de l'atome, et finalement une mesure de $\alpha$ (que l'on peut exprimer en fonction, d'une part, de ce rapport et, d'autre part, de la constante de Rydberg, de la vitesse de la lumière et du rapport de la masse de l'atome à celle de l'électron, bien connues par ailleurs). La comparaison au niveau de $10^{-9}$ en valeur relative de la mesure de $\alpha$ ainsi effectuée, à la valeur extraite de façon indirecte de l'anomalie du rapport gyromagnétique de l'électron, permet de réaliser le test le plus contraignant de la théorie de l'électrodynamique quantique, à l'heure actuelle.

Une mesure de la constante de gravitation $G$ peut également être déduite de l'accélération différentielle de deux nuages d'atomes en chute libre et séparés verticalement. En utilisant de façon simultanée les mêmes faisceaux lasers pour mesurer l'accélération des deux nuages, il est possible de déduire, de la différence des signaux des deux interféromètres, l'effet de l'attraction gravitationnelle d'un élément massif situé 
\〉

entre eux. Les premières expériences ont donné des résultats au niveau de $10^{-3}$ en valeur relative sur la mesure de $G$ [5] ; elles laissent espérer atteindre rapidement le même niveau que les expériences utilisant des balances de torsion $\left(10^{-5}\right)$, ce qui permettra de confronter des mesures obtenues à l'aide de technologies radicalement différentes.

L'utilisation de gravimètres atomiques pourrait également jouer un rôle important dans la redéfinition du kilogramme, qui est la dernière unité du système international (SI) définie par un artefact macroscopique. Suivant l'évolution moderne de redéfinition des unités SI à partir de constantes fondamentales, il est proposé d'utiliser une expérience dite de "balance du watt" pour définir le kilogramme à partir de la constante de Planck $h$. Dans l'une des deux étapes de cette expérience, en cours de réalisation au Laboratoire National de Métrologie et d'Essais ${ }^{(3)}$, la force de pesanteur exercée sur une masse (nominalement de $1 \mathrm{~kg}$ ) est compensée par la force de Laplace subie par une bobine, parcourue par un courant placé dans un champ magnétique. La bobine étant liée mécaniquement à la masse, les mesures électriques permettent de relier la masse à la constante de Planck, via les effets fondamentaux que sont l'effet Josephson et l'effet Hall quantique, pourvu que l'on connaisse par ailleurs de façon exacte et en temps réel l'accélération de la pesanteur locale, g. Dans le cadre de l'expérience française de balance du watt, cette mesure est réalisée à l'aide d'un interféromètre atomique utilisant des atomes de rubidium refroidis par laser (encadré 2). Lorsqu'ils sont lâchés, les atomes tombent le long de la verticale, direction suivant laquelle sont appliquées les trois impulsions laser. Cet instrument, bien qu'encore en phase de développement, atteint aujourd'hui une exactitude relative de quelque $10^{-9}$, comparable à l'état de l'art, qui devrait être bientôt dépassé.

\section{Applications en géophysique}

L'utilisation de gravimètres de très grande exactitude dépasse largement celle de la balance du watt, puisqu'elle ouvre un domaine d'application très prometteur pour les interféromètres atomiques en géophysique. Ces mesures permettent aux géophysiciens d'avoir accès aux anomalies de gravité, et donc d'améliorer la compréhension de la structure interne de notre planète [6]. En effet, le gravimètre atomique est un instrument permettant de mesurer de façon très fine les variations de l'accélération de la pesanteur, à la fois dans le temps et en fonction de la position (encadré 2). Par rapport aux gravimètres conventionnels, fondés sur la mesure de la chute d'un objet macroscopique, ce type d'instrument ne comporte pas de pièces mobiles sujettes à une usure ou une déformation. L'utilisation d'interféromètres atomiques ouvre la possibilité de nouveaux instruments plus transportables. Par ailleurs, l'utilisation de gravimètres atomiques est également envisagée en recherche pétrolière et minière, pour la gestion des ressources aquifères ou le suivi des montées magmatiques précédant une éruption volcanique.

\section{Tests de la gravité}

Les difficultés rencontrées dans les modèles théoriques de grande unification, et les observations récentes en astronomie, semblent indiquer que les lois de la relativité générale sont incomplètes. Il est donc nécessaire de réaliser des tests expérimentaux des lois de la gravitation à différentes échelles, afin de contraindre au maximum les différents modèles. La très grande sensibilité des capteurs inertiels fondés sur l'interférométrie atomique, et leur capacité à réaliser des mesures exactes, en font d'excellents candidats pour ces tests. Différents types d'expériences sont
Les interféromètres atomiques permettent de mesurer de façon exacte l'accélération due à la pesanteur, g. L'unité utilisée ici est le $\mu \mathrm{Gal}$ $\left(1 \mathrm{Gal}=1 \mathrm{~cm} / \mathrm{s}^{2}\right.$ et $1 \mu \mathrm{Gal}=10^{-8} \mathrm{~m} / \mathrm{s}^{2} \approx 10^{-9} \mathrm{~g}$ ). Les données de gauche, enregistrées à Trappes (Yvelines) pendant quatre jours consécutifs, représentent les fluctuations de $g$ autour de la valeur moyenne de $980890742 \mu \mathrm{Gal}$ (chaque point représente une moyenne sur une durée de $\left.2^{\prime} 45^{\prime \prime}\right)$. Ces fluctuations, liées aux marées lunisolaires, sont comparées à un modèle géophysique (courbe bleue).

Ces appareils sont extrêmement sensibles et détectent les ondes sismiques générées par des tremblements de terre provenant de l'autre côté de la Terre, comme celui survenu le 7 octobre 2009 près des îles de Santa Cruz dans le Pacifique Sud (voir la courbe de droite, où l'on a représenté le signal brut de l'interféromètre atomique, sans effectuer de moyenne).

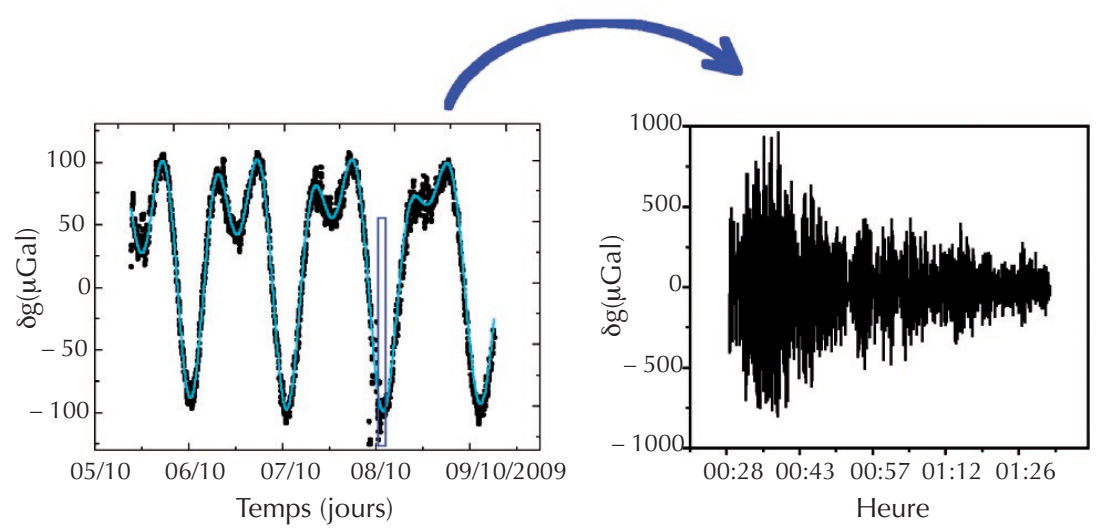

Heure 
en cours de réalisation ou sont envisagés, allant d'échelles microscopiques à des distances comparables à celles du système solaire.

Par exemple, le développement en cours de nouveaux types d'interféromètres atomiques permettra de tester avec une sensibilité sans précédent les lois de la gravitation à courte distance, entre un atome et un corps macroscopique. Ces interféromètres sont réalisés avec les mêmes méthodes de séparatrices atomiques, non plus avec des atomes en chute libre, mais avec des atomes piégés par laser au voisinage d'une surface, à des distances atome-surface de l'ordre du micromètre.

Par ailleurs, des tests de la gravité à grande distance sont envisagés, notamment à travers des expériences dans l'espace. L'apesanteur est un environnement très prometteur pour ce type de tests, puisque les atomes chutent librement en même temps que le satellite, permettant d'augmenter très largement le temps de mesure et donc la sensibilité des interféromètres, qui croît comme le carré du temps d'interaction. Plusieurs projets de tests de la relativité ont déjà été proposés, notamment à l'Agence Spatiale Européenne depuis 2000. Par exemple, le projet SAGAS (Search for Anomalous Gravitation using Atomic Sensors) se propose de rechercher des anomalies de la gravité à très grande distance, en envoyant des capteurs atomiques aux confins du Système solaire. D'autres propositions portent sur un test du principe d'équivalence "faible ", qui postule que la masse inertielle et la masse gravitationnelle sont égales, quel que soit le corps qui tombe (encadré 3).

\section{Conclusion}

Le développement de l'interférométrie atomique a créé un nouveau domaine de mesures de précision concernant, d'une part, les forces d'inertie (gravité, accélération et rotation) et, d'autre part, des grandeurs de physique fondamentale $(\alpha, G$, test de la gravitation...). D'autres applications suivront sans aucun doute, comme le développement de détecteurs d'ondes gravitationnelles atomiques. Une qualité essentielle pour ces applications provient de la capacité à effectuer des mesures de grande stabilité et exactitude. Ces propriétés sont clairement liées à l'utilisation de sources lasers très bien contrôlées en fréquence pour le refroidissement des atomes, et surtout pour la réalisation des séparatrices de l'interféromètre. Mais déjà une nouvelle génération d'expériences se présente, grâce au développement de sources atomiques cohérentes et, notamment, des lasers à atomes, qui sont l'équivalent pour les atomes du laser pour la lumière [1]. On peut donc espérer en physique atomique un succès similaire à ceux liés à l'arrivée du laser en optique, il y a maintenant cinquante ans.

(1) À noter qu'avec des atomes froids (voir [1], l'article de M. Leduc et P. Lemonde dans ce même numéro), on peut atteindre des longueurs d'onde de de Broglie beaucoup plus grandes, de l'ordre du micromètre.

(2) Cette sensibilité à la vitesse de rotation est bien connue en optique : c'est l'effet Sagnac.

(3) Le Laboratoire National de Métrologie et d'Essais est un établissement public, chargé notamment du pilotage général de la métrologie française.

\section{Références}

1 - M. Leduc et P. Lemonde, «Atomes froids : réseaux optiques et horloges », dans ce numéro, p. 46.

2 • Ch. J. Bordé, Physics Letters, A140 (1989) 10-12.

3 - F. Pereira Dos Santos et A. Landragin, Physics World, 20 (2007) 32-37.

4 P. Cladé et al., Phys. Rev. Lett., 96 (2006) 033001.

5 • J.B. Fixler et al., Science, 315 (2007) 74-77.

$6 \bullet$ M. Diament, « Mesure du champ de pesanteur terrestre ", Techniques de l'Ingénieur, Mesures mécaniques et dimensionnelles, R 1814 (2005).

\section{Test du principe d'équivalence "faible "}

Un test du principe d'équivalence "faible » peut être effectué en mesurant la différence entre les accélérations subies par deux espèces atomiques différentes, toutes deux en chute libre. Ce test serait réalisé en orbite proche de la Terre, pour bénéficier du champ de gravité terrestre et de la très grande sensibilité des interféromètres dans l'espace, offerte par l'utilisation de très grands temps d'interaction. Des études expérimentales préliminaires sont en cours, notamment dans le cadre du projet ICE, qui est une collaboration entre I'Institut d'Optique, I'ONERA et l'Observatoire de Paris, soutenue par le CNES. L'expérience est installée dans un avion « zéro-g » (photographie de gauche), qui réalise des vols paraboliques permettant d'obtenir des périodes d'apesanteur de plus de vingt secondes durant lesquelles est testée I'expérience. La figure de droite montre les franges d'interférences atomiques pendant les phases zéro-g (G. Stern et al., Eur. Phys. J. D, 53 (2009) 353). On a représenté le nombre normalisé d'atomes dans un des ports de sortie, en fonction du déphasage lié à l'accélération résiduelle évaluée à l'aide d'un accéléromètre macroscopique. Les franges ne sont pas visibles en présence de gravité.
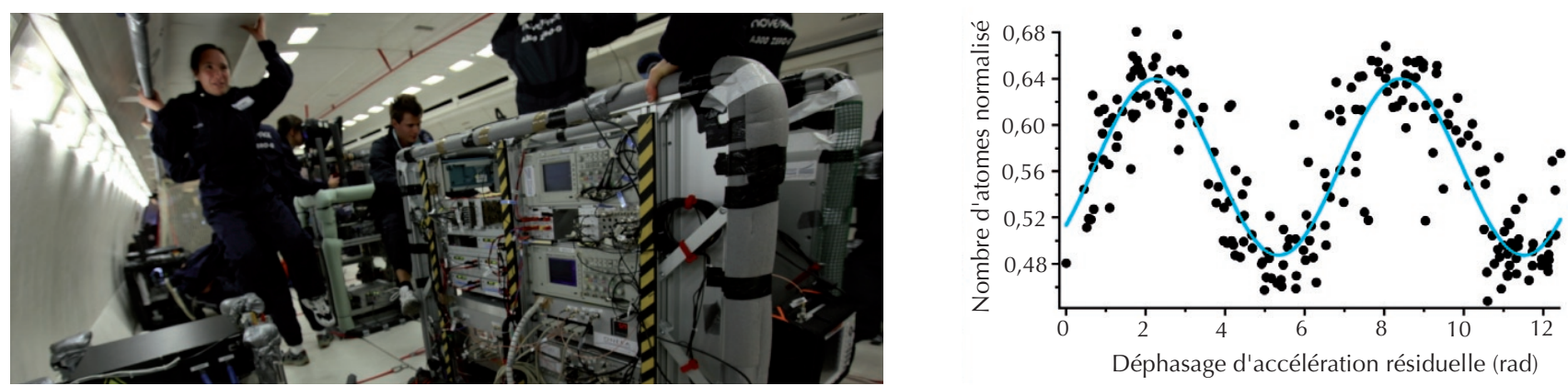